\section{Importancia del lapso desde el último parto para el pronóstico del cáncer de mama primario}

Un primer parto temprano y un gran número de partos confieren a la mujer un bajo riesgo de cáncer de mama. Sin embargo, varios estudios recientes muestran que el embarazo a término tiene un efecto dual, pues aumenta ese riesgo transitoriamente después del parto para luego reducirlo a largo plazo. Aduciendo que un cáncer establecido durante el embarazo o antes puede acelerar su crecimiento bajo la influencia de la alta concentración de hormonas, un grupo de investigadores daneses estudió la influencia del lapso desde el último parto en la supervivencia postoperatoria. Aprovechando los extensos registros nacionales disponibles en $\mathrm{Di}$ namarca, el estudio retrospectivo se basó en datos sobre cáncer de mama primario en 5652 mujeres que tenían hasta 45 años de edad en el momento del diagnóstico. Los registros contenían información detallada sobre los diagnósticos, características de los tumores, regímenes de tratamiento, factores reproductivos y estadísticas vitales de todas las mujeres. En $90 \%$ de la población estudiada el tratamiento quirúrgico primario fue mastectomía total más excisión de tejidos axilares y, en el resto, extirpación del tumor y disección axilar. Según el programa danés de tratamiento del cáncer vigente en el momento de la cirugía, cada paciente se clasificó de alto o bajo riesgo por criterio histopatológico. Las pacientes de bajo riesgo tenían tumores de $<5 \mathrm{~cm}$ en diámetro sin metástasis a los ganglios linfáticos axilares y sin invasión de la piel ni de la línea de resección profunda. Las mujeres premenopáusicas con ganglios negativos tenían tumores clasificados como de grado histológico I. Las que se clasificaron de alto riesgo tenían tumores primarios de $>5 \mathrm{~cm}$ o con metástasis a los ganglios axilares o invasión de la piel o de la línea de resección profunda. Las pacientes premenopáusicas con anaplasias de grado II y III se clasificaron también de alto riesgo. Las pacientes con cáncer de mama bilateral, metástasis distantes, cáncer inflamatorio o con contraindicaciones al tratamiento postoperatorio planeado o que no fueron tratadas según las normas quirúrgicas no se asignaron a protocolos de tratamiento.

Las pacientes de bajo riesgo no recibieron tratamiento sistémico posquirúrgico en ninguno de los programas. De 1978 a 1982, las de alto riesgo se asignaban a radioterapia postoperatoria o a radio- terapia y tratamiento sistémico. En el programa de 1983 a 1989, se asignaban a tratamiento sistémico y radioterapia o solo a tratamiento sistémico. La radioterapia posmastectomía estaba dirigida a la pared torácica y a los ganglios linfáticos regionales (axilares, supraclaviculares, infraclaviculares y paraesternales). En el programa en marcha desde 1990 hasta ahora, las pacientes de alto riesgo reciben tratamiento sistémico de acuerdo con el estado de receptores de la hormona esteroide. Se administra radioterapia si el tumor invade la línea de resección profunda. Todas las pacientes con tumorectomías se someten a radioterapia del tejido residual del pecho.

Hubo una supervivencia bruta de 5 años en $58,7 \%$ de las mujeres diagnosticadas en los dos primeros años después del parto y una supervivencia de 10 años en $46,1 \%$, frente a proporciones respectivas de $78,4 \%$ y $66,0 \%$ en mujeres que tuvieron su último parto más de 2 años antes del diagnóstico de cáncer. Un embarazo reciente tuvo un efecto negativo tanto en las que recibieron tratamiento adyuvante como en las que no lo tuvieron. En las mujeres con un parto reciente ( $<2$ años) clasificadas de bajo riesgo y por lo tanto sin tratamiento adyuvante, la supervivencia bruta fue de $75,0 \%$ (5 años) y de $55,6 \%$ (10 años), comparada con $88,5 \%$ y $77,8 \%$ en mujeres cuyo último parto fue más de 2 años antes del diagnóstico. Después de ajustes por la edad, los antecedentes reproductivos y el estadio de la enfermedad, un diagnóstico emitido menos de 2 años después del último parto se asoció con una supervivencia significativamente menor (riesgo relativo: 1,58; IC95\%: 1,24 a 2,02) que la de las mujeres que habían tenido su último parto más de 5 años antes. Otros análisis indicaron que este efecto no era modificado por la edad al diagnóstico ni por el tamaño del tumor o estado de los ganglios.

En mujeres jóvenes en general y en embarazadas y lactantes en particular es difícil diagnosticar el cáncer por la densidad de las mamas, lo que se refleja en una importante demora del diagnóstico. En el estudio presentado, la tendencia de las mujeres que estuvieron embarazadas poco tiempo antes a tener enfermedad más avanzada podría hasta cierto punto relacionarse con esa demora. Sin embargo, se pudo ajustar por ese factor. De manera que, independientemente de la demora diagnóstica, un parto reciente significó un aumento del riesgo de morir de alrededor de $60 \%$, frente a las demás 
mujeres con cáncer de mama. La explicación más admisible de este fenómeno parece ser que el embarazo cambia el curso de la enfermedad porque aumenta el riesgo de que se presente un patrón de crecimiento sumamente maligno en células tumorales ya existentes. Es, pues, importante tener en cuenta los partos anteriores al orientar a las mujeres jóvenes con cáncer de mama, ya que un parto reciente debe considerarse un factor pronóstico negativo. (Kroman N, Wohlfahrt J, Andersen KW, Mouridsen HT, Westergaard T, Melbye M. Time since childbirth and prognosis in primary breast cancer: population based study. Br Med J 1997;315:851-855.)

\section{Revisión del Reglamento Sanitario Internacional}

La OMS está revisando las normas del Reglamento Sanitario Internacional (RSI) con el fin de adaptarlas al presente volumen del tráfico y el comercio internacionales, teniendo en cuenta las tendencias actuales en la epidemiología de las enfermedades transmisibles y la amenaza de las enfermedades emergentes. El RSI revisado proporcionará un mecanismo para la notificación inmediata de todos los brotes de enfermedades que exijan atención urgente a nivel internacional. Para agilizar el proceso, los brotes se caracterizarán inicialmente en función del síndrome clínico en vez del diagnóstico preciso. Según el Reglamento, un brote deberá notificarse solamente si cumple dos criterios: corresponder a la definición de caso de uno de los síndromes especificados y constituir un acontecimiento que exija atención inmediata en el ámbito internacional. Una vez notificado el brote, la OMS tomará la decisión de hacerlo público en consulta con el Estado Miembro afectado. Si una investigación ulterior indica que el brote no tiene importancia desde el punto de vista internacional, se hará un declaración pública al respecto. Según el RSI revisado, no será necesario notificar más los brotes habituales de enfermedades endémicas. Por lo tanto, el cólera no será notificable a menos que el brote ocurra en una zona donde no es endémico, se trate de un serotipo nuevo, sea de gravedad singular o provoque restricciones de viaje o comercio por parte de otros Estados Miembros.

Con las definiciones de caso correspondientes a los síndromes se procura asegurar que todos los brotes de enfermedades transmisibles que representen un peligro internacional sean notificables en función del nuevo Reglamento. Los síndromes propuestos para notificación son fiebre hemorrágica aguda, síndrome respiratorio agudo, síndrome dia- rreico agudo, ictericia aguda y síndromes neurológicos agudos, así como una categoría que incluye otros síndromes notificables de origen infeccioso. La notificación de un síndrome será normalmente seguida de un informe sobre la enfermedad específica en cuanto se confirme el diagnóstico. Cuando el diagnóstico se conoce, puede notificarse la enfermedad en vez del síndrome correspondiente. Los países determinarán si un brote debe notificarse o no, de acuerdo con los criterios médicos, epidemiológicos y operacionales siguientes: alto riesgo de propagación a escala internacional; tasa de letalidad anormalmente elevada; aparición desusada; síndrome recientemente reconocido; gran interés por parte de los medios de comunicación; o riesgo de que se impongan restricciones a los viajes o al comercio.

Los cambios del RSI no afectarán de modo alguno las actividades regulares de vigilancia y notificación de enfermedades a la OMS. El RSI revisado no suplantará esas actividades ni interferirá con ellas. Se está llevando a cabo un estudio piloto en ciertos países para evaluar la rapidez y facilidad de la forma de notificación por síndrome. También se han realizado seminarios en cada región de la OMS para dar a conocer los cambios propuestos. El nuevo texto, en preparación por un pequeño grupo de expertos en salud pública y ley internacional, se enviará a principios de 1998 a los Estados Miembros y a las organizaciones interesadas para suscitar comentarios. (World Health Organization. Revision of the International Health Regulations: Progress report, January 1998. Wkly Epidemiol Rec 1998;73: 17-19.)

\section{Las causas de muerte sospechadas y las establecidas posmórtem en pacientes con sida}

El sida se ha convertido en una de las causas principales de muerte, especialmente de jóvenes adultos entre los 25 y 40 años de edad. Datos de la OMS indican que en todo el mundo más de 150 personas mueren diariamente a causa del sida. Sin embargo, desde que se reconoció el síndrome, la proporción de exámenes posmórtem en pacientes que mueren de sida en los Estados Unidos de América ha disminuido de 46 a 17\%. Las autopsias de esos pacientes muestran una discrepancia sustancial con los diagnósticos clínicos en cuanto a la causa de muerte. Esta discrepancia es mucho mayor que en la población en general. Sin embargo, en el estudio de las enfermedades infecciosas, las autopsias siguen desempeñando un papel importante para comprender la epidemiología, la fisiopatología y el 
diagnóstico de enfermedades como el sida y las infecciones emergentes por arenavirus, virus de Ebola y virus Hanta. Varios estudios sobre los resultados de las autopsias muestran que puede haber discrepancia entre los diagnósticos clínicos y posmórtem hasta en $72 \%$ de los casos. Para elucidar este fenómeno, un grupo de investigadores de la Universidad Federal de Uberlandia, Minas Gerais, Brasil, revisó los archivos y los informes de las necropsias de 52 pacientes que murieron de sida en el hospital universitario durante un período de 7 años y compararon los resultados del examen posmórtem con los diagnósticos premórtem. Se recopilaron datos sobre la edad, sexo, factores de riesgo de infección por $\mathrm{VIH}$, manifestaciones clínicas y resultados de las pruebas de laboratorio de los pacientes fallecidos. Todos los incluidos en el estudio satisfacían los criterios para el diagnóstico de sida diseminados por los Centros para el Control y la Prevención de Enfermedades, Atlanta, EUA. Se encontraron diagnósticos clínicos confirmados por pruebas de laboratorio, otros que se sospechaban a juzgar por la respuesta al tratamiento y otros que se sospechaban pero que no pudieron confirmarse. Las pruebas histopatológicas de órganos y tejidos incluyeron tinción específica para el diagnóstico de infecciones oportunistas, como la tinción de ZiehlNeelsen para micobacterias, la de ácido-Schiff periódico (PAS), la de Gomori-Grocott para hongos, y las técnicas inmunohistoquímicas para la identificación de Trypanosoma cruzi, Toxoplasma gondii y linfomas. En siete de los 52 pacientes la necropsia se había hecho solo parcialmente y no incluyó todos los órganos. Se consideró que había concordancia entre los diagnósticos clínico y posmórtem cuando eran iguales, aunque se hubieran descubierto durante la necropsia otros trastornos no sospechados.

Los pacientes murieron a edades que oscilaban entre los 15 y 54 años de edad (media de 33,3 \pm 9,9 años); 36 eran varones; 16, toxicómanos; 9, homosexuales; 6 , heterosexuales; 4 , homosexuales toxicómanos; y 1, hemofílico. En 16 de los pacientes no se determinó ningún factor de riesgo de infección por VIH. En dos de los siete que tuvieron exámenes posmórtem parciales, no se encontró ningún indicio de infección oportunista o neoplasia. Estos se excluyeron del análisis de concordancia o discrepancia entre los diagnósticos pre y posmórtem. De los 50 pacientes analizados, 23 tuvieron por lo menos un diagnóstico clínico confirmado por autopsia; es decir, hubo concordancia en $46 \%$. Hubo 24 pacientes $(48 \%)$ que tuvieron como mínimo una enfermedad relacionada con el sida que no se sospechaba debido a ausencia de manifestaciones clínicas. En 26 de los pacientes (52\%) la autopsia permitió diagnosticar más de una enfermedad. Solo se había tenido sospecha clínica de 27 de los 89 diagnósticos hechos posmórtem.

El hallazgo más común por autopsia fue la infección por citomegalovirus en 16 casos, de los cuales ninguno se había sospechado. En 16 casos se diagnosticó micosis sistémica. También hubo seis casos de histoplasmosis, seis de criptococosis (cinco diagnosticados premórtem) y cuatro de candidiasis. Se encontraron 11 casos de toxoplasmosis (nueve del sistema nervioso central [SNC] solamente, uno del SNC y el esófago y un caso diseminado). De estos casos de toxoplasmosis solo se habían diagnosticado cinco, cuya infección se limitaba al SNC. Nueve pacientes tuvieron infección diseminada por micobacterias, sospechada en solo tres de ellos. De tres casos de enfermedad de Chagas reactivada, uno tenía miocarditis y meningoencefalitis, uno miocarditis solamente y uno meningoencefalitis solamente. Se encontró enfermedad neoplásica en cinco casos, tres de ellos con linfoma diseminado, uno con adenocarcinoma del intestino delgado y uno con sarcoma de Kaposi diseminado. Otras enfermedades encontradas en la autopsia que no se sospechaban incluyeron seis casos de tuberculosis, tres de neumocistosis, tres de candidiasis, tres de encefalitis necrotizante y uno de herpes simple. El estudio mostró la presencia simultánea de múltiples infecciones oportunistas que contribuyeron a la muerte de pacientes de sida, muchas de ellas no detectadas en vida. Los autores concluyen que los pacientes de sida deben someterse a exámenes minuciosos para detectar la presencia de infecciones y cánceres, especialmente si no responden a tratamientos dirigidos a estados patológicos reconocidos. (Borges AS, Ferreira MS, Nishioka S de A, Silvestre MTA, Silva AM, Rocha A. Agreement between premortem and postmortem diagnoses in patients with acquired immunodeficiency syndrome observed at a Brazilian teaching hospital. Rev Ins Med Trop São Paulo 1997;39:217-221.)

\section{Otra enfermedad emergente: la úlcera de Buruli}

La OMS ha anunciado que se hará un esfuerzo internacional a modo de coalición contra la úlcera de Buruli, enfermedad terrible que afecta principalmente a la gente joven, especialmente en África. Desde 1948 se han notificado casos en Benín, Costa de Marfil, Gabón, Ghana y Uganda. Como verdadera enfermedad emergente, es poco conocida la úlcera de Buruli, infección micobacteriana asociada con el ambiente húmedo del trópico que destruye los tejidos produciendo lesiones sumamente graves en las extremidades. La enfermedad 
parece estar sustituyendo a la lepra como flagelo de la sociedad en lugares como Costa de Marfil, donde se han registrado 400 casos desde 1978.

Hay muchas preguntas sin respuesta en lo que se refiere al agente de la enfermedad, Mycobacterium ulcerans, las condiciones necesarias para su supervivencia en el ambiente natural y los mecanismos de transmisión al ser humano. Se piensa estudiar a fondo el aumento reciente de casos de úlcera de Buruli desde el punto de vista de la posible influencia de la vacuna BCG y del papel que pueda desempeñar en ello la epidemia de infección por VIH y sida. Actualmente no hay ningún tratamiento médico para controlar eficazmente la úlcera de Buruli, que ocupa el tercer lugar, después de la tuberculosis y la lepra, entre las enfermedades micobacterianas más ampliamente propagadas del mundo. Hasta ahora, el único tratamiento posible consiste en extensas excisiones de las lesiones seguidas de injertos de piel. Este procedimiento no solo es largo y costoso, sino que deja secuelas. La enfermedad existe en focos endémicos en los lugares pantanosos de las zonas tropicales y subtropicales de Asia, Oceanía, América Latina y África. (World Health Organization. WHO joins battle against a new emerging disease, Buruli ulcer. Press Release WHO/88, 8 December 1997.)

\section{La Comisión de Derechos Humanos aprobó una resolución sobre la alimentación}

En su 53a sesión celebrada en Ginebra, Suiza, del 10 de marzo al 18 de abril de 1997, la Comisión de Derechos Humanos de las Naciones Unidas aprobó una resolución sobre el derecho a la alimentación. En el preámbulo de dicha resolución se recuerda la Declaración Universal de Derechos Humanos, en la que se expresa que todas las personas tienen el derecho a un estándar de vida adecuado para la salud y el bienestar propio y de su familia, incluida la alimentación. Se citan además la Declaración Universal sobre la Erradicación del Hambre y la Malnutrición; las disposiciones del Convenio Internacional sobre Derechos Económicos, Sociales y Culturales en las que se reconoce el derecho fundamental de cada persona de satisfacer el hambre; la Declaración de Roma sobre Seguridad Alimentaria y el Plan de Acción de la Cumbre Mundial de la Alimentación, de 1996. En la resolución se reconoce que los problemas del hambre y de la inseguridad alimentaria son de alcance mundial. Debido al crecimiento esperado de la población y la mayor demanda de los recursos naturales, es probable que esos problemas sean no solo duraderos sino cada vez mayores en algunas regiones, a menos que se inicien acciones concertadas y urgentes. También se llama la atención a que un ambiente político, social y económico de paz y estabilidad es un fundamento esencial para que los Estados otorguen la prioridad adecuada a la seguridad alimentaria y la erradicación de la pobreza. Se reitera, como en la Declaración de Roma, que los alimentos nunca deben usarse como instrumento de presión política o económica. En este sentido se reafirma la importancia de la cooperación y la solidaridad internacional y la necesidad de evitar medidas unilaterales que no estén de acuerdo con la legislación internacional y la Carta de las Naciones Unidas y que pongan en peligro la seguridad alimentaria. Cada Estado debe adoptar una estrategia afín con sus recursos y capacidad para lograr sus metas individuales al ejecutar las recomendaciones de la Declaración y el Plan de Acción de Roma y, a la vez, prestar cooperación regional e internacional con el fin de contribuir soluciones colectivas a los problemas alimentarios de un mundo cada vez más interrelacionado por sus instituciones, sociedades y economías.

La resolución luego manifiesta que el hambre constituye un ultraje y una violación de la dignidad humana y, por lo tanto, requiere la adopción de medidas urgentes para eliminarla a nivel nacional, regional e internacional. Todas las personas tienen derecho a un abastecimiento de alimentos seguros y nutritivos coherente con el derecho de librarse del hambre para desarrollarse plenamente y conservar su capacidad mental y física. Es intolerable que más de 800 millones de personas en todas partes del mundo y especialmente en países en desarrollo no tengan suficiente comida para satisfacer sus necesidades nutricionales básicas, situación que viola sus derechos humanos fundamentales.

Es, por lo tanto, necesario movilizar y lograr la óptima asignación y utilización de recursos técnicos y financieros de diverso origen, incluido el alivio de la carga de la deuda externa de los países en desarrollo, para reforzar las actividades nacionales en la ejecución de políticas encaminadas a lograr una seguridad alimentaria sostenible. La Comisión alentó al Comité de Derechos Económicos, Sociales y Culturales a prestar atención en sus actividades a los derechos reconocidos en el artículo 11 del Convenio Internacional. Se suscribió también a lo solicitado en el Plan de Acción de la Cumbre Mundial de la Alimentación dirigido al Alto Comisionado para los Derechos Humanos. Dicho Plan pide que este, en consulta con los suscritos al tratado y en colaboración con organismos especializados y programas del sistema de las Naciones Unidas, mecanismos intergubernamentales y organizaciones no guberna- 
mentales, defina más claramente los derechos relacionados con la alimentación en el artículo 11 del Convenio Internacional sobre Derechos Económicos, Sociales y Culturales, y proponga formas de hacer respetar esos derechos para lograr los compromisos y objetivos de la Cumbre Mundial de la Alimentación, teniendo en cuenta la posibilidad de que se formulen voluntariamente directrices para lograr la seguridad alimentaria para todos. Por último, la Comisión invitó al Alto Comisionado para los Derechos Humanos a informar sobre la ejecución de la resolución en la 54a sesión de la Comisión de Derechos Humanos de las Naciones Unidas. (World Health Organization. United Nations Commission on Human Rights adopts resolutions and decisions on health-related matters. Int Dig Health Legis 1997;48:429-430.)

\section{Triatómidos infectados con Trypanosoma cruzi en Cuernavaca (Morelos), México}

La infección por Trypanosoma cruzi en el ser humano, que se conoce como enfermedad de Chagas, es una parasitosis de gran importancia en América Latina. Fue originalmente una enfermedad enzoótica, pero a medida que se invadieron y degradaron los ecosistemas y se ahuyentaron los animales salvajes, sus vectores naturales, chinches reduvídeos del género Triatoma, se adaptaron a alimentarse de los seres humanos y animales domésticos. La enfermedad se convirtió así en una zoonosis típica. En México, el índice de infección por T. cruzi en los triatómidos capturados varía notablemente entre un estado y otro. Se han encontrado infectadas $94 \%$ de las muestras de Triatoma rubida en Sonora; $96 \%$ y 90\%, respectivamente, de las muestras de T. phyllosoma mazzottii y T. phyllosoma pallidipennis en Guerrero; de 33 a $66 \%$ de las de T. phyllosoma pallidipennis en Morelos y $50 \%$ de las de la misma especie en Michoacán; 14,2\% de las muestras de $T$. dimidiata en Yucatán; $62 \%$ de las de T. barberi en Jalisco; $50 \%$ de las de T. phyllosoma en Aguascalientes y $17,9 \%$ de las de T. barberi en Oaxaca. La infección natural por T. cruzi se encontró en $31,8 \%$ de los especímenes de T. pallidipennis capturados en Jojutla, Morelos. También se han encontrado reservorios en zonas urbanas. Debido a la captura previa en las zonas rurales del estado de Morelos de triatómidos con la infección natural por T. cruzi, se estudió la frecuencia de vectores infectados en la zona urbana de Cuernavaca.

Se dieron charlas a los padres en escuelas locales sobre la importancia de las enfermedades parasitarias y de ese modo se obtuvo su ayuda en la captura de insectos en las casas y sus alrededores durante 18 meses. Se les enseñó a los voluntarios cómo evitar infectarse accidentalmente y a diferenciar a los vectores de los reduvídeos fitófagos. La identificación de las especies se llevó a cabo en el laboratorio.

En esta búsqueda se hallaron dos tipos de triatómidos: T. pallidipennis y T. barberi. En las inmediaciones de las casas se capturaron $1035 \mathrm{~T}$. pallidipennis adultos, de los cuales $88 \%$ tenían flagelados de Trypanosoma spp., y 25 T. barberi adultos, $70 \%$ de los cuales estaban igualmente infectados. Estos insectos se capturaron en 24 localidades diferentes. Las zonas urbanizadas en donde se capturaron los insectos tienen espacios sin construcción y barran$\cos$ que constituyen buenos hábitats. En nueve lugares, de 90 a $95 \%$ de los triatómidos capturados estaban infectados con T. cruzi; en 11 barrios, la infección osciló entre 81 y $88 \%$; en tres, entre 74 y $76 \%$. El índice más bajo correspondió al centro urbano: cinco triatómidos infectados de 15 captura$\operatorname{dos}(33 \%)$.

A pesar de los altos índices de infección con $T$. cruzi, los especímenes de T. pallidipennis tuvieron una baja densidad de tripomastigotes en las heces. Por otra parte, estos insectos tienen patrones de defecación prolongados, factor con un efecto sumamente importante en la capacidad vectorial de los triatómidos. Teniendo en cuenta ambos factores, T. pallidipennis no es actualmente un buen vector de T. cruzi y la transmisión a las personas sería fortuita. No obstante, los altos porcentajes de infección natural muestran que es necesario estimular el estudio de la situación de la enfermedad de Chagas en el territorio y aumentar la cantidad de información que tiene al respecto la población, como medida para prevenir esa parasitosis. (Cortés-Jiménez M, Nogueda-Torres B, Alejandre-Aguilar R, IsitaTornelli L, Ramírez-Moreno E. Frequency of triatomines infected with Trypanosoma cruzi collected in Cuernavaca City, Morelos, Mexico. Rev Latinoam Microbiol 1996;38:115-119.) 